\title{
Systemic Lupus Erythematosus and the broken dental tool
}

\author{
Brittany Urso', Katelyn M. Updyke', James A. Solomon ${ }^{1,2,3}$ \\ ${ }^{1}$ University of Central Florida College of Medicine, Orlando Florida, USA, ${ }^{2}$ University of Illinois College of Medicine, \\ Urbana Illinois, USA, ${ }^{3}$ Ameriderm Research, Ormond Beach Florida, USA
}

Corresponding author: Brittany Urso, E-mail: Brittany.Urso@knights.ucf.edu

Systemic Lupus Erythematosus (SLE) is an autoimmune condition which can be exacerbated by many factors, many of which are unknown. While flares can be controlled with medications, we often are unsure of their cause which makes disease maintenance difficult. It is possible that investigation of a patient's habits, lifestyle, and diet may provide clues which make disease maintenance, and even remission, possible.

We present a 40-year-old female with a medical history of Systemic Lupus Erythematosus (SLE) associated with contact allergies to nickel, and drug reactions to penicillin, tetracycline, clindamycin, erythromycin, and sulfa. Her first SLE episode occurred following a weekend at a tomato harvest and subsequent episodes followed upper respiratory tract symptoms treated with antibiotics. These episodes resolved spontaneously. A dentist breaking a stainless steel tool, lodging the tip within her tooth, precipitated 2 years of chronic fatigue, joint pains, butterfly rash, peripheral edema, irritable bowel, and general malaise. Her ANA titer was $>$ 1:640 and homogeneous suggesting a drug eruption.

On physical exam, the patient had a butterfly rash and urticarial papules and plaques on her trunk and extremities in a distribution corresponding to areas in contact with metal.

Detailed history of her first SLE episodes were associated with ingesting dozens of tomatoes as well as with taking aspirin with antibiotics. To investigate her allergies, a lymphocytic activation assay was performed under laminal flow hoods, using RPMI media without bovine serum, penicillin or streptomycin. The assay revealed lymphocyte activation to $10^{-9}-10^{-18}$ molar
Table 1: Foods containing high amounts of salicylates, the foods listed are common sources of salicylates [1]

\begin{tabular}{ll}
\hline Foods containing higher amounts of salicylates \\
\hline Aspirin & Curry powder \\
Tomatoes & Paprika \\
Berries & Thyme \\
Dried fruits & Garam masala \\
Rosemary & Tea \\
Licorice candy & Peppermint candy \\
\hline
\end{tabular}

Table 2: Common sources of nickel within food, foods cooked within stainless steel cookware, such as those listed, are sources of food containing nickel [2]

Common sources of nickel within food

Food cooked using stainless steel cookware

Flash frozen vegetables

Pasteurized milk products

Fresh, non-Kosher chicken

to penicillin, salicylates, nickel, cobalt, chrome, and sulfonamide. Additionally, the reaction to salicylates was at $10^{-9}$ molar but was $10^{-18}$ molar to Bayer Aspirin ${ }^{\text {TM }}$ tablet; (n.b.: the tablet combines salicylates with metal fillers).

The patient noted significant improvement with dietary restriction of salicylate and nickel containing foods, including tomatoes, as well as avoidance of nickel containing cookware, which not only includes base metal cookware but also stainless steel (Table 1 and 2) $[1,2]$. Photosensitivity resolved when she avoided food sources of psoralens. Additionally, removal of the dental tool fragment and metal restorations (multiple stainless steel root canal crowns) was associated with complete remission. As result, through careful analysis of the patient's habits, lifestyle, and diet, we were able to determine and control triggers, such as salicylates, 
nickel, and drugs which induce photosensitivity, which precipitated her SLE flare.

\section{REFERENCES}

1. Swain AR, Dutton SP, Truswell AS. Salicylates in foods. J Am Diet Assoc. 1985;85:950-60.

2. Kamerud KL, Hobbie KA, Anderson KA. Stainless steel leaches nickel and chromium into foods during cooking. J Agric Food Chem. 2013;61:9495-501.

Copyright by Brittany Urso, et al. This is an open-access article distributed under the terms of the Creative Commons Attribution License, which permits unrestricted use, distribution, and reproduction in any medium, provided the original author and source are credited.

Source of Support: Nil, Conflict of Interest: None declared. 